\title{
Production of allelochemicals in Fischerella muscicola under different environmental conditions
}

\author{
Elisabeth M. Gross, Eric v. Elert and Friedrich Jüttner
}

\section{Introduction}

The existence of allelopathic interactions among phytoplankton species remains controversial (for review see R1CE 1984). Even if allelochemicals can be isolated, their action in the ecosystem, e.g. on phytoplankton seasonal succession, remains debatable. The question is whether they are released or actively excreted into the environment and what concentrations are necessary to be effective in the waterbody. A major problem relates to the large distances water-soluble allelochemicals have to move to reach target organisms in the phytoplankton communities (LEW1s 1986). On the other hand, reports on the isolation of allelochemicals from benthic cyanobacteria have been published in recent years (MAsON et al. 1982, Moore et al. 1984).

We studied the allelopathic potential of the benthic cyanobacterium Fischerella muscicola, that strongly inhibits other cyanobacteria (FLORES \& WOLK 1986) and to a lesser extent members of the chlorophytes and diatoms. We isolated the main active compound by a bioassay directed extraction procedure, purified it and called it fischerellin. Results of the physical and chemical characteristics of fischerellin and its physiological role have been published recently (GRoss et al. 1991).

Studying allelopathic interactions in benthic cyanobacteria is promising for several different reasons: many cyanobacteria are known to produce a great number of different secondary metabolites (MoORE 1981). Such compounds are often investigated for pharmaceutical purposes, although they may also have an ecological and physiological role. Additionally, in benthic and edaphic habitats allelopathy seems to be a powerful competitive strategy because of the proximity of adjacent species. We suggest that certain allelochemicals are transmitted by direct contact of cells for which purpose lipophilic inhibitors of low molecular weight would be very suitable. As F. muscicola is able to kill algae growing adjacently, we studied the excretion and transfer of fischerellin.

In this context it is also important that the quality and quantity of allelochemicals produced might depend on environmental conditions, e.g. the strength of competition, nutrient depletion or shading (BOYER et al. 1987). Accordingly, we studied fischerellin production by $F$. muscicola experimentally under nitrate, phosphate and light limitation.

\section{Materials and methods}

Culture conditions for Fischerella were the same as described previously (Gross et al. 1991). Nutrient and light limitation experiments were performed with WCmedium (GUILLARD \& LORENZEN 1972) in $300 \mathrm{ml}$ aerated tubes (compressed air enriched to $0.2 \% \mathrm{CO}_{2}, 25^{\circ} \mathrm{C}$ ). For P-limitation $1 \mu \mathrm{M} \mathrm{KH}_{2} \mathrm{PO}_{4}$ was used and the medium was supplemented with $40 \mu \mathrm{M} \mathrm{KCl}$. Nitrate depleted cultures received $10 \mu \mathrm{M} \mathrm{NaNO}$. Light limitation was achieved by covering the tubes with black nylon stockings (20den) which reduced light intensity from 60 to $10 \mu \mathrm{E} \cdot \mathrm{m}^{-2} \cdot \mathrm{s}^{-1}$. Axenic cultures semicontinuously diluted were kept for six weeks. Half of the culture suspension was replaced with fresh medium every fourth day. Each treatment was run in duplicate. The cells were harvested by centrifugation, frozen and lyophilized. The methanolic extracts were concentrated and subjected to reversed phase HPLC analysis $(250 \cdot 4.6 \mathrm{~mm}$ RP C18 column, Kromasil, $5 \mu \mathrm{m}$, EKA Nobel, Sweden). UV-detection of fischerellin at $267 \mathrm{~nm}$ was achieved with a Beckman System Gold Diode Array Detection Module 168 (Beckman, Munich, FRG).

Fischerellin excretion was investigated with precleaned and sterilized XAD-16-beads (Serva, Heidelberg, FRG) which were added to culture suspensions of $F$. muscicola. At the end of the experiments (1 week or 6 weeks), the culture suspension was separated into cyanobacterial cells, XAD beads and culture filtrate by centrifugation $(10 \mathrm{~min}, 900 \cdot \mathrm{g})$ and filtration (Whatman GF/F). XAD beads and cyanobacterial lyophilisate were extracted with methanol, the extract was concentrated and subjected to HPLC. The culture filtrate was assayed for fischerellin as described previously (Gross et al. 1991).

\section{Results and discussion}

\section{Effect of environmental conditions on fischerellin production}

There are few reports on the effect of environmental conditions on the production of secondary metabolites in cyanobacteria. The content of geosmin, a terpenoid volatile compound, varied with culture condition in Fischerella muscicola (WU \& 


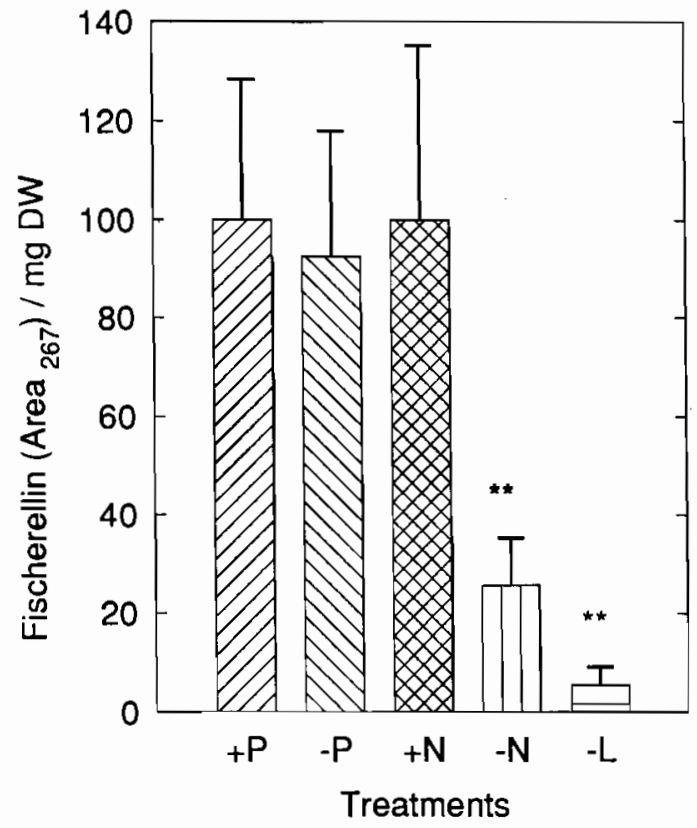

Fig. 1. Fischerellin production in F. muscicola under different nutrient and light conditions. The relative amount of fischerellin produced was calculated as $\%$-value of the control. Vertical error bars indicate the $99 \%$ confidence intervalls. The stars indicate that the nitrogen and light limited treatments are significant different from the other treatments at the $0,1 \%$ level (Bonferroni multiple range test).

JÜTTNER 1988). The synthesis of geosmin was dependent on the growth rate rather than on nutrient depletion. In contrast, the toxin content of Protogonyaulax tamarensis, a marine dinoflagellate, increased dramatically at the beginning of the stationary growth phase under P-limited conditions, but declined gradually under N-limited conditions (Boyer et al. 1987).

In contrast to the results of BOYER et al. (1987), fischerellin production of $F$. muscicola when calculated per unit biomass did not increase in P-limited cells (Fig. 1). The P-depleted cultures reached only $40 \%$ of the biomass and $40 \%$ of chlorophyll per unit biomass compared to non-limited cultures. Per unit chlorophyll, however, P-limited cells produced more than twice the amount of fischerellin. Thus P-limited Fischerella appears to invest the same energy per unit biomass for the production of the allelochemical although its chlorophyll content is low and metabolic activity presumably depressed.
Nitrate depletion was expected to have no effect on fischerellin production because of the nitrogen-fixing capacity of Fischerella. Surprisingly, a sharp and significant decline of fischerellin production resulted under conditions of both nitrate depletion and light limitation (Fig. 1). Given that nitrogen fixation provides a competitive advantage, there may be no need for the production of so much inhibitor. Comparing the results from nitrate depletion and light limitation, energy costs seem to be responsible for the decrease of inhibitor production. Nitrate depleted cultures reached only $75 \%$ of the biomass of the controls, light limited only 5-10\%. Both cultures might have been energy limited; weak light reduces the energy load by decreasing photophosphorylation, and nitrogen fixation involves high ATP-consumption. Furthermore, as fischerellin contains at least two nitrogen atoms in its molecule, the synthesis of this metabolite may be affected by nitrate depletion.

In summary, neither phosphorus, nitrate nor light limitation results in an increased production of fischerellin per unit biomass. In contrast, energy shortage caused by nitrate depletion or light limitation leads to a dramatic decrease of the cellular contest of fischerellin.

\section{Excretion and transfer of fischerellin}

In neither the present limitation experiments nor in previous experiments did we detect fischerellin in the culture filtrate. Modelling the possible mechanism of transfer of allelochemicals in benthic habitats, we suggest the transfer of these compounds to adjacent cells by direct contact (Gross et al. 1991). Fischerellin is very well suited to pass cell membranes due to its lipophilic nature and low molecular weight. To test this hypothesis, Fischerella was grown in batch culture on a rotary shaker together with XAD-16 adsorber beads with a lipophilic surface and a diameter of $1.6 \mathrm{~mm}$. The beads were used here to simulate other cells that might grow together with F. muscicola. At the end of the experiments, lasting 1 or 6 weeks, the culture suspension was separated into cyanobacterial cells, beads and culture filtrate and these fractions were tested for fischerellin. No inhibitor was found in the culture filtrate, but a considerable amount of fischerellin had bound to the beads. These results support the former hypothesis that the inhibitor is transmitted by direct contact to adjacent cells. To quantify the amount of released fischerellin and to exclude any effects from lysed 
and dead cells, further experiments with continuous cultures are in preparation.

The co-culturing of cyanobacteria with lipophilic adsorber beads represents a suitable and interesting model system to investigate the exchange of allelochemicals between adjacent cells in benthic habitats.

\section{Acknowledgement}

This work was supported by Volkswagen Stiftung (I/63 733).

\section{References}

Boyer, G. L., Sullivan, J. J., Andersen, R. J., Harrison, P. J. \& TAYLOR, F. J. R., 1987: Effects of nutrient limitation on toxin production and composition in the marine dinoflagellate Protogonyaulax tamarensis. - Mar. Biol. 96: 123-128.

Flores, E. \& Wolk, C. P., 1986: Production, by filamentous, nitrogen-fixing cyanobacteria, of a bacteriocin and of other antibiotics that kill related strains. - Arch. Microbiol. 145: 215-219.

Gross, E. M., WOLK, C. P. \& Jütner, F., 1991: Fischerellin, a new allelochemical from the freshwater cyanobacterium Fischerella muscicola. - J. Phycol. 27: $686-692$.

Guillard, R. R. L. \& Lorenzen, C. J., 1972: Yellowgreen algae with chlorophyllidec. - J. Phycol. 8: $10-14$.
LEW/S Jr., W. M., 1986: Evolutionary interpretation of allelochemical interactions in phytoplankton algae. - Am. Nat. 127: 184-194.

Mason, C. P., Edwards, K. R., Pignatello, J., Carison, R. E., Gleason, F. K. \& WoOD, J. M., 1982: Isolation of chlorine-containing antibiotic from the freshwater cyanobacterium Scytonema bofmanni. - Science 215: 400-402.

Moore, R. E., 1981: Constituents of Blue-green Algae. - In: Scheurer, P. J. (ed.): Marine Natural Products: 1-52. - Academic Press, Inc., Orlando, Florida, USA.

Moore, R. E., Blackman, A. J., Cheuk, C. E. \& MrnDERSE, J. S., 1984: Hapalindoles: New alkaloids from the blue-green alga Hapalosiphon fontinalis. - J. Am. Chem. Soc. 106: 6456-6457.

Rrce, E. L., 1984: Allelopathy. 2nd Ed., Chapter 6. Academic Press, Inc., Orlando, Florida, USA.

WU, J. T. \& JUtTNER, F., 1988: Effect of environmental factors on geosmin production by Fischerella muscicola. - Wat. Sci. Tech. 20: 143-148.

Authors' address:

Elisabeth M. Gross and Eric v. Elert, MPI of Limnology, Dept. Ecophysiology, D-24302 Plön, Germany.

F. JUTTNER, Institute of Plant Biology/Limnology, University of Zürich, CH-8802 Kilchberg, Switzerland. 